\title{
ECG Signal Analysis for Troponin Level Assessment and Coronary Artery Disease Detection: the NEEDED Study 2014
}

\author{
Dominika Długosz, Aleksandra Królak \\ Łódź University of Technology, \\ Faculty of Electrical, Electronic, Computer, \\ and Control Engineering, Institute of Electronics, \\ ul. Wólczańska 211/215, 90-924 Łódź, Poland, \\ Email: dominika.a.m.dlugosz@gmail.com, \\ aleksandra.krolak@p.lodz.pl
}

\author{
Trygve Eftestøl, Stein Ørn, Tomasz Wiktorski, \\ Kay Raymond Jenssen Oskal, Martin Nygård \\ University of Stavanger, \\ Faculty of Science and Technology, \\ Department of Electrical and Computer Engineering, \\ 4036 Stavanger, Norway, \\ Email: \{trygve.eftestol, stein.orn, tomasz.wiktorski\}@uis.no, \\ \{martin.a.nygard, kay.oskal\}@gmail.com
}

\begin{abstract}
Physical exercise is widely recognized as beneficial to the cardiovascular system. However, intense exercise may also carry fatal risk. Investigation of this phenomenon is one of the primary purposes of the North Sea Race Endurance Exercise Study (NEEDED). This paper describes analysis of electrocardiograms (ECG) and heart rate signals collected from amateur athletes, participants of the race, to facilitate noninvasive estimation of the level of cardiac troponin I (cardiovascular risk biomarker) and detection of coronary artery disease (CAD). It was demonstrated that the combination of ECG and heart rate parameters can predict CAD with high specificity (up to $98 \%$ ) and relatively good sensitivity. Moreover, while troponin level assessment is unlikely to be reliably performed using regression techniques, it might be possible using a new, probabilistic classification-based model. Further evaluation of the latter requires the use of additional data, which is one of possible directions for the future work.
\end{abstract}

\section{INTRODUCTION}

C ARDIAC troponins $\mathrm{T}$ and $\mathrm{I}(\mathrm{cTnT}, \mathrm{cTnI})$ are protein subunits involved in contraction of the heart muscle. Their increased blood levels are widely associated with an occurrence of damage to the myocardium of diverse etiologies, including coronary artery disease (CAD) [1], [2], [3]. Nonetheless, recent studies have reported increase in cTnT and cTnI levels incident to prolonged, high-intensity physical exercise in presumably healthy individuals, predominantly recreational athletes [4]-[9].

This phenomenon was observed also in the frame of the North Sea Race Endurance Exercise Study (NEEDED) conducted at the University of Stavanger and Stavanger University Hospital in Norway [10], [11]. Aiming to explore the impact of long-term physical effort on the physiology of the cardiovascular system, the study recruited its participants from recreational cyclists competing in Nordsjørittet (the North Sea Race) - an annual cycling competition, organized in Rogaland, Norway. In 2014, over a thousand study subjects were examined i.a. for blood levels of cardiovascular biomarkers (including cTnI) and electrocardiograms (ECG). Supplementary data was retrieved from some of the participants' sports watches. Herein described research work was concerned with analysis of the abovementioned data. The main hypothesis stated that blood level of cTnI and presence of CAD can be predicted based on parameters of ECG and heart rate (HR). In particular, the prediction might be guided by physical effort-induced changes in not explicitly pathological ECG.

\section{STUdy POpUlation AND THE DATASET}

The investigated population comprised 160 presumably healthy individuals. A total of 53 individuals were assessed by coronary computed tomography angiography, 6 of whom were diagnosed with CAD. In these 6 and further 14 cases, at least one cTnI level was elevated (>190 ng/l [10]). The analyzed data included:

- blood levels of cTnI, measured at: $24 \mathrm{~h}$ before, $3 \mathrm{~h}$ after, and $24 \mathrm{~h}$ after the race;

- 10-second 12-lead ECG recordings, collected at the same measuring time points as the cTnI data;

- clinical data: participants' age and BMI;

- formerly processed [12] HR data from sports watches.

\section{DATA PREPARATION}

\section{A. ECG preprocessing and segmentation}

The ECG was preprocessed by: filtering (band-pass Butterworth infinite impulse response filter, order: 5, cut-off frequencies: 1 and $40 \mathrm{~Hz}$ ) and baseline offset removal. Subsequently, the signal was segmented into heartbeat templates using tools implemented in BioSSPy toolbox. Key points of the ECG (vertices, onsets, and endpoints of $\mathrm{P}, \mathrm{S}$, and $\mathrm{T}$ waves) were determined based on averaged beat templates from lead I similarly to our previous work [13]. Onsets of positive waves $(\mathrm{P}, \mathrm{T})$ were searched for within a window preceding the maximal ascending slope of the wave $\left(n_{m s}\right)$ : $\left[n_{m s}-w, n_{m s}-s\right]$, with $w$ - window size, $s$ - additional spacing to compensate for a possible 'M pattern'. Onset candidates were points $n \in\left[n_{m s}-w, n_{m s}-s\right]$ satisfying conditions:

$$
N_{c}=\left\{n:\left|y^{\prime}(n)\right| \leqslant\left|k \cdot y^{\prime}\left(n_{m s}\right)\right| \wedge y^{\prime \prime}(n) \geqslant 0\right\}
$$


TABLE I: Parameters of detection of $\mathrm{M}$ and $\mathrm{W}$ patterns.

\begin{tabular}{|c|c|c|c|c|}
\hline Wave & Pattern & Lead & k & I \\
\hline P & M & I, II & 0.2 & 3 \\
\hline R & M and W & V1, V5, V6 & 0.2 & 2 \\
\hline S & W & I, V6 & 0.3 & 2 \\
\hline
\end{tabular}

where: $y$ - the signal; $k$ - a threshold factor. Selection criterion, depending on point type, was of form:

$$
\begin{aligned}
& \text { 1) } \arg \min _{n \in N_{c}} g(n), g(n)=\left|y(n) \cdot y^{\prime}(n)\right| \text {, or } \\
& \text { 2) } \arg \max _{n \in N_{c}} y^{\prime \prime}(n)
\end{aligned}
$$

Detection $\mathrm{P}$ and $\mathrm{T}$ waves endpoints was performed analogically on the descending slopes of the waves. For $\mathrm{S}$ wave (negative wave) endpoint, the respective segment was inverted. The parameters: $w, s$, and $k$ were tuned separately for each point type based on standard durations of the waveforms.

\section{FEATURE EXTRACTION}

\section{A. ECG signal}

ECG features described below were defined in three areas: time domain, frequency domain, and correlation analysis.

1) Features from lead-I signal measurements: basic timedomain features, including:

- heart rate,

- QT interval duration corrected for HR (according to the formula of Fridericia [14]),

- ST segment duration,

- ST elevation,

- P wave shape coefficient, i.e. the ratio of width and amplitude of the wave.

2) $M$ and $W$ patterns: notches in $\mathrm{P}, \mathrm{R}$, and $\mathrm{S}$ waves ${ }^{1}$ (see Fig. 1). An $M$ pattern was deemed to be present in the signal if a sufficient number $l$ of samples $n$ of given wave satisfied the following criteria:

$$
\left\{\begin{array}{l}
y^{\prime}(n)<0 \wedge y(n)>k \cdot y\left(n_{\max }\right) \quad \text { for } n_{\text {on }}<n<n_{\max } \\
y^{\prime}(n)>0 \wedge y(n)>k \cdot y\left(n_{\max }\right) \quad \text { for } n_{\max }<n \leqslant n_{\text {end }}
\end{array}\right.
$$

where: $n_{\text {on }}, n_{\text {peak }}, n_{\text {end }}$ - indices of onset, peak, and endpoint of the given waveform; $y$ - signal values; $k$ - threshold factor: minimal portion of the peak amplitude. For detection of $\mathrm{W}$ patterns, the signal was inverted. Parameters $k$ and $l$ were determined empirically for each wave type, as summarized in table I.

3) Heart axis: direction ${ }^{2}$ estimated using a pair of perpendicular leads: I and aVF:

$$
\theta=\arctan \left(\frac{V_{n e t, a V F}}{V_{n e t, I}}\right)
$$

\footnotetext{
${ }^{1}$ Depending on wave and lead of their manifestation, $\mathrm{M}$ and $\mathrm{W}$ patterns might be symptoms of atrial hypertrophy, left or right bundle branch block, and other conditions [15].

${ }^{2}$ The mean direction of the electric field vector (in the coronal plane) throughout an ECG cycle. Its significant deviation may be a symptom of disorders affecting the conduction system (e.g. bundle branch block) [16]
}

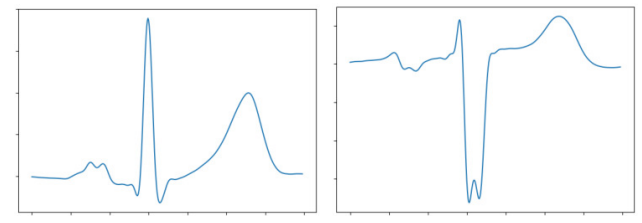

Fig. 1: Exemplary averaged beat templates: (left) $M$ pattern in a lead-I $\mathrm{P}$ wave; (right) $\mathrm{W}$ pattern in a lead-V1 R wave.

$V_{\text {net }}$ denotes net QRS potential in a lead, calculated as:

$$
V_{\text {net }}=\left\{\begin{array}{l}
\max \left(V_{Q R S}\right) \quad \text { if } \min \left(V_{Q R S}\right) \geqslant 0 \\
\max \left(V_{Q R S}\right)-\left|\min \left(V_{Q R S}\right)\right| \text { otherwise }
\end{array}\right.
$$

where $V_{Q R S}(t)$ - voltage of the QRS complex [17].

4) Lead-I QRS templates correlation: used to assess internal morphological consistency of a recording. The parameter was calculated as the mean value of the upper-triangle elements (excluding the diagonal) of correlation matrix of QRS templates from a single lead-I signal.

5) Frequency-domain features: derived from power spectra of the QRS complexes. QRS-only signal was extracted by a mask: QRS selection windows (rectangular with half-Gaussian slopes), replicated at locations of the $\mathrm{R}$ peaks. Parameters derived from power spectrum of the masked signal included:

- two slopes characteristic for log-transformed lowfrequency moiety of the signal, calculated over frequency ranges: [3 Hz, $8 \mathrm{~Hz}$ ] and [15 Hz, $19 \mathrm{~Hz}$ ] (as in [18]),

- the mean of power spectral density signal over frequency range $[150 \mathrm{~Hz}, 300 \mathrm{~Hz}]$ (upper half of the range).

\section{B. Heart rate}

HR signal from sports watches, resampled and processed in the previous works, was a base for calculation of two parameters:

1) HRp99: mean of 99th percentile HR samples:

$$
H R_{p 99}=\frac{\overline{\left\{h r \in H R: h r \geqslant P_{99}(H R)\right\}}}{H R_{\max }}
$$

where: $H R_{\max }$ - maximal predicted age-dependent $\mathrm{HR}$ : $H R_{\max }=208-0.7 \cdot$ Age [19]

2) HR90 time: a portion of the race time in which participant's HR was above $90 \%$ their individual HR reserve:

$$
t_{H R 90}=\frac{\sum_{i=1}^{n} i\left[H R_{i}>\left(H R_{\text {rest }}+0.9 \cdot H R_{\text {reserve }}\right)\right]}{\sum_{i=1}^{n} i}
$$

where: $\mathrm{n}$ - the number of samples, $H R_{\text {rest }}$ - resting HR (from ECG prior to the race), and $H R_{\text {reserve }}$ - individual HR reserve: $H R_{\text {reserve }}=H R_{\text {max }}-H R_{\text {rest }}$ [4].

\section{Clinical data and parameters from the previous work}

- participants' age and BMI,

- max and mean HR from Tinghaug hill segment (a major steep climb of the race, associated with substantial effort and strain to the heart) [12], normalized by $H R_{\max }$.

Following the hypothesis on the importance of changes versus momentary state, parameters from days 2 and 3 were 
expressed as ratio (for HR) or difference with respect to day 1. To sum up, there were: 14 parameters from the ECG (each day), 4 from the HR, and 2 from the clinical data - in total, 48 features. A general assumption was to use data gathered prior to, or simultaneously with collection of blood for troponin assay. Thus, there were three sets of input parameters for cTnI level assessment, with 16, 34, and 48 features. CAD detection was performed on two sets: one with the 48 features and the second, additionally including cTnI levels (51 features).

\section{EXPERIMENTS SETUP}

The data analysis had two main objectives: cTnI level estimation (separately for each day) and CAD prediction together, four problems. The small size and strongly nonuniform distribution of the data motivated the use of leave-one-out cross-validation (LOOCV) approach.

\section{A. CAD detection}

Two methods were applied for this binary classification task:

1) Automatically optimized classification: a search for the best classifier launched with the use of TPOT - genetic programming-based software providing tools for model selection and tuning [20]. The number of generations and population size were set to 7 and 70 respectively.

2) Grid-search-optimized decision tree classifier: the classifier was optimized in terms of i.a. class weights and maximal depth. The best estimator instance was passed to LOOCV loop.

The results were evaluated using two types of metrics: confusion matrix and the area under the receiver operating characteristic (ROC) curve, weighed by counts of true positive (TP) and true negative (TN) observations.

\section{B. cTnI level estimation}

Due to a strong bias towards the lowest values and vast dispersion of the highest readings, the cTnI values were logtransformed before analysis. Estimation of the cTnI level - as a continuous variable - was approached using two techniques:

1) Automatically optimized regression approach: determination of an optimal regression model with the TPOT software (7 generations and population size of 70).

2) Probabilistic classification-based approach: an intermediate class definition was obtained by stratification of the logtransformed cTnI levels for a given day into 2 to 10 layers of equal breadth. Test samples were classified using a logistic regressor with probabilistic classification output. Next, in the LOOCV scheme, the cTnI level for each test sample was estimated by computing a weighted average of the class centers (medians, $\mathbf{c}_{\mathbf{c T n I}}$ ) with class probabilities vector $\mathbf{p}$ as weights:

$$
c T n I=10^{\left(\mathbf{p}^{\mathbf{T}} \cdot \mathbf{c}_{\mathbf{c T n I}}\right)}
$$

\section{RESULTS AND DISCUSSION}

\section{A. CAD detection}

The results of CAD prediction are summarized in Table II. Results were influenced by a random component (randomly seeded decision tree classifier; obtaining a deterministic output is possible, but might introduce a bias to the results). The
TABLE II: Summary of the results of CAD prediction; TP = true positive, $\mathrm{TN}=$ true negative, $\mathrm{FP}=$ false positive, $\mathrm{FN}=$ false negative (given in counts of observations).

\begin{tabular}{|c|c|c|c|c|}
\hline Dataset & Approach & $\begin{array}{c}\text { Model } \\
\text { specification }\end{array}$ & $\begin{array}{c}\text { Confusion } \\
\text { matrix }\end{array}$ & $\begin{array}{c}\text { ROC } \\
\text { area }\end{array}$ \\
\hline $\begin{array}{c}\text { Without } \\
\text { cTnI data }\end{array}$ & $\begin{array}{c}\text { TPOT } \\
\text { optimization }\end{array}$ & $\begin{array}{c}\text { gradient boost. } \\
\text { classifier }\end{array}$ & $\begin{array}{c}\text { TN 154, FP 0, } \\
\text { FN 6, TP 0 }\end{array}$ & 0.42 \\
\cline { 2 - 5 } & $\begin{array}{c}\text { Decision tree } \\
\text { + grid search }\end{array}$ & $\begin{array}{c}\text { number } \\
\text { of features: 5 }\end{array}$ & $\begin{array}{c}\text { TN: 152, FP: 2, } \\
\text { FN: 3, TP: 3 }\end{array}$ & 0.74 \\
\hline \multirow{2}{*}{$\begin{array}{c}\text { With } \\
\text { cTnI data }\end{array}$} & $\begin{array}{c}\text { TPOT } \\
\text { optimization }\end{array}$ & $\begin{array}{c}\text { gradient boost. } \\
\text { classifier }\end{array}$ & $\begin{array}{c}\text { TN 154, FP 0, } \\
\text { FN 6, TP 0 }\end{array}$ & 0.83 \\
\cline { 2 - 5 } & $\begin{array}{c}\text { Decision tree } \\
\text { + grid search }\end{array}$ & $\begin{array}{c}\text { number } \\
\text { of features: 5 }\end{array}$ & $\begin{array}{c}\text { TN: 151, FP: 3, } \\
\text { FN: 1, TP: 5 }\end{array}$ & 0.91 \\
\hline
\end{tabular}

information on the true cTnI levels significantly influenced the results, in particular in terms of the false positive (FP) detections. When the cTnI data was included, all FP results were from the elevated cTnI group; otherwise, all FPs belonged to the low cTnI class.

Formerly [12], CAD detection based on the HR and clinical data with the best true positive rate (TPR) of 0.86 (6/7 cases) and the same true negative rate (TNR). Herein described experiments were performed with a greater scope of parameters (from the ECG signal), but with reduced number of CAD-positive observations (by one sample, i.e. 14\%). TPR decreased to 0.5 (3/6 cases). However, TNR was noticeably higher, reaching 0.98 (152/154 cases). With the cTnI data, TPR increased to 0.83 (a single undetected case). Most significant factor contributing to TPR decrease is the depletion of the positive class strength.

The results do not unambiguously prove that features derived from the ECG signal improve CAD prediction rate (compared to HR only). However, they clearly demonstrate the value of ECG in excluding otherwise suspected CAD in healthy individuals.

\section{B. Troponin level determination}

1) Regression approach: this approach failed to establish a reliable prediction model. In none of the cases, the scores reached positive values (while 1 denotes a perfect prediction, and 0 - a constant model). One of possible explanations lies in the physiology of the circulatory system: the differences in ECG and HR parameters between the individuals did not sufficiently correlate with differences in cTnI level over the range of the values. Indeed, it should not be assumed that e.g. the duration of the QT segment would increase proportionally to the increase in the extent of CAD, associated with elevation in cTnI. The negative conclusion was confirmed by an independent team at the University of Stavanger [21].

2) Probabilistic classification-based approach: exemplary results (for day 3) are presented in Fig. 2. The highest true value was excluded from the plot to provide a better plot scale for analysis of the results. The estimation was strongly biased towards the lowest level, containing majority of the observations. Additionally, there are some false high predictions. However, there is a group of observations actually 

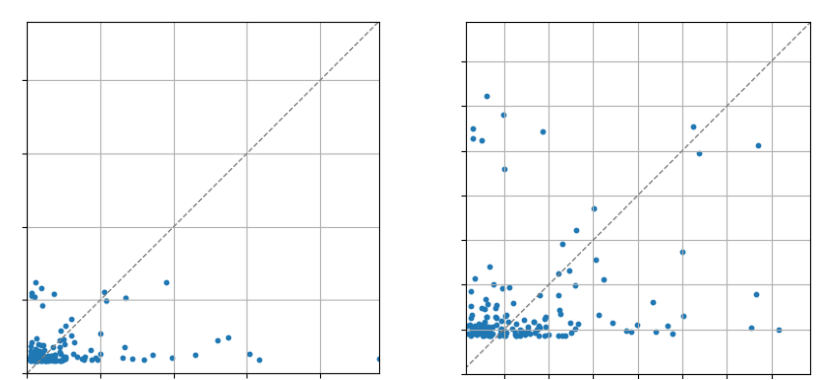

Fig. 2: cTnI level determination with probabilistic classification: results for day 3; horizontal: true, vertical: predicted values; (left) full set, (right) zoom at the lower range.

following the diagonal of the plot. Although they constituted minority of the set, these predictions were fairly accurate.

There were multiple factors hindering the prediction. The number of samples (160) was low. This effect was escalated by data imbalance. In day-1 data, $39 \%$ of the samples had the same cTnI level of $1.6 \mathrm{ng} / \mathrm{l}$, and $86 \%$ did not exceed the level of $5 \mathrm{ng} / \mathrm{l}$ (mean \pm standard deviation: $2.2 \pm 0.8$ ). In the remaining $14 \%$ of the dataset, the values spread between 4.9 and $284.8 \mathrm{ng} / \mathrm{l}$ (mean \pm standard deviation: $26.9 \pm 55.8$ ). On the remaining days, there were additional substantial, but isolated peaks $(5025.9 \mathrm{ng} / \mathrm{l}$ for day 2 and $7918.5 \mathrm{ng} / \mathrm{l}$ for day 3 - the latter being more than 33-fold larger than any other in the set). Excluding a single high-cTnI sample (in LOOCV) further amplified the imbalance.

\section{CONCLUSIONS}

The herein reported project was concerned with analysis of data from the NEEDED research program with two main objectives. First of all, the level of circulating cTnI following prolonged, strenuous exercise was to be estimated using information derived from ECG and HR signals. The main conclusion to this problem was negative, though meaningful for the future research: the correlation between cTnI and parameters derived from HR and ECG was not found sufficient to establish a continuous, regression-based model for estimation of the level of the former. However, it is possible that the alternative classification-based approach is more likely to correspond to the underlying physiological mechanisms governing the exercise-induced cTnI response. Nevertheless, both conclusions need to be validated on a greater dataset.

The second goal was improvement of the rate of detection of CAD compared to previously achieved results. ECG was not found to increase the sensitivity of the prediction. However, this refers to a decrease in TP detections by 1 observation with lowered strength of the positive class. On the other hand, the ECG features notably enhanced the detection in terms of its specificity - from $86 \%$ to $98 \%$. Best results of prediction of $\mathrm{CAD}$ could be achieved by including the cTnI level information into the analysis. The two types of information appear to be complementary - cTnI improves sensitivity of the model (a single undetected case), while ECG and HR data promote its specificity by effectively excluding non-CAD cases of cTnI elevation.

\section{REFERENCES}

[1] J. Sarko and C. V. Pollack, "Cardiac troponins," Am J Emerg Med, vol. 23, no. 1, pp. 57-65, Jul. 2002.

[2] L. Babuin and A. S. Jaffe, "Troponin: the biomarker of choice for the detection of cardiac injury," CMAJ, vol. 173, no. 10, pp. 1191-1202, Nov. 2005.

[3] T. Omland, M. A. Pfeffer, S. D. Solomon, J. A. de Lemos, H. Røsjø et al., "Prognostic Value of Cardiac Troponin I Measured With a Highly Sensitive Assay in Patients With Stable Coronary Artery Disease," J Am Coll Cardiol, vol. 61, no. 12, pp. 1240-1249, Mar. 2013.

[4] P. Aagaard, A. Sahlén, L. Bergfeldt, and F. Braunschweig, "Heart Rate and Its Variability in Response to Running-Associations with Troponin," Med Sci Sports Exerc, vol. 46, no. 8, p. 1624, Aug. 2014.

[5] R. Shave, A. Baggish, K. George, M. Wood, J. Scharhag et al., "Exercise-Induced Cardiac Troponin Elevation: Evidence, Mechanisms, and Implications," J Am Coll Cardiol, vol. 56, no. 3, pp. 169-176, Jul. 2010.

[6] T. M. H. Eijsvogels, M. D. Hoogerwerf, M. F. H. Maessen, J. P. H. Seeger, K. P. George et al., "Predictors of cardiac troponin release after a marathon," J Sci Med Sport, vol. 18, no. 1, pp. 88-92, Jan. 2015.

[7] A. Legaz-Arrese, K. George, L. E. Carranza-García, D. MunguíaIzquierdo, T. Moros-García et al., "The impact of exercise intensity on the release of cardiac biomarkers in marathon runners," Eur J Appl Physiol, vol. 111, no. 12, pp. 2961-2967, Dec. 2011.

[8] S. Regwan, E. A. Hulten, S. Martinho, J. Slim, T. C. Villines et al., "Marathon Running as a Cause of Troponin Elevation: A Systematic Review and Meta-Analysis," J Interv Cardiol, vol. 23, no. 5, pp. 443450, Oct. 2010.

[9] E. Serrano-Ostáriz, A. Legaz-Arrese, J. L. Terreros-Blanco, M. LópezRamón, D. Cremades-Arroyos et al., "Cardiac Biomarkers and Exercise Duration and Intensity During a Cycle-touring Event," Clin J Sport Med, vol. 19, no. 4, pp. 293-299, Jul. 2009.

[10] Ø. Skadberg, Ø. Kleiven, M. Bjørkavoll-Bergseth, T. Melberg, R. Bergseth et al., "Highly increased Troponin I levels following high-intensity endurance cycling may detect subclinical coronary artery disease in presumably healthy leisure sport cyclists," Eur J Prev Cardiol, vol. 24, no. 8, pp. 885-894, May 2017.

[11] Ø. Kleiven, M. Bjoerkavoll-Bergseth, Ø. Skadberg, T. Melberg, B. Auestad et al., "P3242prolonged release of cardiac troponin I after endurance exercise could indicate silent coronary artery disease in recreational athletes," Eur Heart J, vol. 38, no. suppl_1, Aug. 2017.

[12] K. Oskal, "Myocardial damage during mountain bike race - an analysis of data from Nordsjørittet 2014 (NEEDED study)," Master's thesis, University of Stavanger, Jun. 2016.

[13] D. Długosz, T. Eftestøl, S. Ørn, T. Wiktorski, and A. Królak, "The North Sea Bicycle Race ECG Project: Time-Domain Analysis," vol. 11. Prague: Annals of Computer Science and Information Systems, Sep. 2017, pp. 1353-1356.

[14] B. Vandenberk, E. Vandael, T. Robyns, J. Vandenberghe, C. Garweg et al., "Which QT Correction Formulae to Use for QT Monitoring?" Journal of the American Heart Association, vol. 5, no. 6, Jun. 2016.

[15] Thomas B. Garcia and Neil E. Holtz, EKG - sztuka interpretacji, 1st ed. Warszawa: MediPage, 2007.

[16] A. C. Guyton and J. E. Hall, Textbook of Medical Physiology, 11th ed. Pennsylvania: ELSEVIER SAUNDERS, 2006.

[17] E. Pietka and J. Kawa, Information Technologies in Biomedicine. Springer Science \& Business Media, May 2010.

[18] M. L. Talbi and A. Charef, "PVC discrimination using the QRS power spectrum and self-organizing maps," Comput Methods Programs Biomed, vol. 94, no. 3, pp. 223-231, Jun. 2009.

[19] H. Tanaka, K. D. Monahan, and D. R. Seals, "Age-predicted maximal heart rate revisited," J Am Coll Cardiol, vol. 37, no. 1, pp. 153-156, Jan. 2001.

[20] R. Olson, R. Urbanowicz, P. Andrews, N. Lavender, L. Kidd et al., "Automating Biomedical Data Science Through Tree-Based Pipeline Optimization," in Applications of Evolutionary Computation: 19th European Conference, EvoApplications 2016, Porto, Portugal, March 30 - April 1, 2016, Proceedings, Part I, ser. Lecture Notes in Computer Science. Springer, Cham, Mar. 2016, pp. 123-137.

[21] M. Bjorkavoll-Bergseth, O. Kleiven, T. Melberg, O. Skadberg, B. Uestad et al., "Increased heart rate does not explain the cardiac troponin increase following strenous exercise - the needed 2014 advanced heart rate monitor substudy," Europrevent, p. 296, 2018. 